\title{
e-Phaïstos
}

e-Phaïstos

Revue d'histoire des techniques / Journal of the history

of technology

VIII-1 | 2020

Écomusée : une expansion internationale

\section{Le réseau d'électricité de Tianjin (Chine), 1900-1960. Histoire et valorisation patrimoniale}

The Electric System of Tianjin (China), 1900-1960: History and Value Enhancement as Heritage

Jiali Huang

\section{(2) OpenEdition}

Journals

Édition électronique

URL : http://journals.openedition.org/ephaistos/7737

DOI : 10.4000/ephaistos.7737

ISSN : 2552-0741

Éditeur

IHMC - Institut d'histoire moderne et contemporaine (UMR 8066)

Référence électronique

Jiali Huang, «Le réseau d'électricité de Tianjin (Chine), 1900-1960. Histoire et valorisation patrimoniale », e-Phaïstos [En ligne], VIII-1 | 2020, mis en ligne le 29 avril 2020, consulté le 06 mars 2021. URL : http://journals.openedition.org/ephaistos/7737 ; DOI : https://doi.org/10.4000/ephaistos 7737

Ce document a été généré automatiquement le 6 mars 2021.

Tous droits réservés 


\section{Le réseau d'électricité de Tianjin (Chine), 1900-1960. Histoire et valorisation patrimoniale}

The Electric System of Tianjin (China), 1900-1960: History and Value Enhancement as Heritage

Jiali Huang

Thèse : références bibliographiques

Jiali HUANG, Le réseau d'électricité de Tianjin (Chine) 1900 à 1960. Histoire et valorisation patrimoniale, Thèse de doctorat en Histoire des techniques, Université Paris 1 Panthéon Sorbonne, Institut d'Histoire Moderne et Contemporaine (UMR 8066), soutenue le 28 mars 2019, un volume de texte (533 p).

\section{Directeur de thèse}

Anne-Françoise GARÇON, Professeur émérite, Université Paris 1 Panthéon Sorbonne

\section{Jury}

Alain BELTRAN, Directeur de recherche CNRS UMR IRICE (président) Jean-François BELHOSTE, Directeur de recherche, EPHE

Giovanni Luigi FONTANA, Professeur des Universités, Université de Padoue (prérapporteur)

Marina GASNIER, McF HDR, Université de Bourgogne Franche-Comté, UTBM Hélène VACHER, Professeur des Universités, École nationale supérieure d'architecture de Nancy (pré-rapporteur)

1 Monsieur le Président, Mesdames et Messieurs, les membres du jury,

2 Je suis très honorée de pouvoir présenter devant vous cette thèse de doctorat d'histoire intitulée : le réseau d'électricité de Tianjin (Chine) 1900 à 1960. Histoire et valorisation patrimoniale. 
3 À proximité de Pékin, Tianjin est la quatrième plus grande ville de Chine. Longtemps appelée Tientsin, elle doit sa grande prospérité à son passé comme port destiné aux commerces interne et international, ainsi qu'à la juxtaposition de neuf concessions étrangères entre 1860 et 1947. L'industrialisation dans Tianjin est donc autant un territoire d'aventures pour les entrepreneurs étrangers qu'un laboratoire pour les pionniers chinois. Elle constitue un centre d'intérêt pour la recherche académique qui s'est focalisée sur les secteurs majeurs que sont le textile et l'industrie chimique. Or, la première ville électrifiée en Chine a été Tianjin. Mais peu d'études ont été consacrées au rôle de l'électrification dans la modernisation de Tianjin. Cela tient à l'historiographie limitée par le cadre académique chinois. L'électrification de Tianjin, pendant la période de réveil de la Chine, était contrôlée par les étrangers. C'est pourquoi les chercheurs chinois ont manifesté peu d'intérêt en faveur de son étude. Ils étaient plutôt motivés par la valorisation de la force patriotique mise en œuvre pour le développement industriel ainsi que l'éducation technique. Aujourd'hui, l'ouverture de la sphère académique est plus forte et je m'inscris dans une historiographie nouvelle et évolutive. En effet, l'absence des Chinois dans la première phase d'électrification de Tianjin ne les a pas empêchés de diriger l'exploitation des centrales électriques après leur nationalisation. J'ai donc souhaité focaliser mon regard sur le transfert technique entre l'Occident et la Chine, transfert qui s'est réalisé progressivement.

4 Le transfert technologique dans le domaine de l'électricité s'est réalisé à partir des pays occidentaux vers la Chine, à l'époque beaucoup moins développée. Nous ne pouvons pas imaginer le choc que le tramway et l'éclairage électriques ont provoqué chez les habitants chinois qui vivaient dans un autre monde technique à défaut d'infrastructures modernes. Pourtant, les échanges entre les deux mondes avaient commencé dès l'Antiquité. C'est la Chine qui est à l'origine de la diffusion de techniques importantes dans le monde occidental, par exemple, la boussole, l'imprimerie etc. Joseph Needham se demandait pourquoi l'industrie n'est pas née en Chine à l'époque moderne alors qu'elle avait devancé l'Occident par ses inventions et innovations techniques depuis plus de 15 siècles. À mon avis, cette approche est incomplète. Un autre volet consiste à savoir comment la Chine a réussi en un siècle à rattraper l'Occident qui l'avait dominée si longtemps dans les domaines technologiques. C'est à cette question que j'ai essayé de répondre dans mon travail.

5 L'étude de l'électrification m'a paru être un bon vecteur, car elle permet de distinguer l'introduction d'une innovation technologique et sa généralisation. Une fois les conditions réunies, l'électricité, énergie invisible, est devenue le fer de lance pour remodeler le paysage industriel de Tianjin en lui permettant de passer d'une ville commerciale à une ville industrielle; ceci tant pour le domaine tangible des infrastructures que pour le domaine intangible des savoirs, c'est-à-dire l'accumulation des connaissances et la formation des ingénieurs chinois. C'est pourquoi j'ai décidé de traiter de l'évolution du réseau électrique, depuis sa domination par les étrangers à partir de 1900 jusqu'en 1960. L'analyse de cette période clé m'a permis de déchiffrer l'acquisition d'une capacité en innovation technologique, ce qui a finalement conduit à un tournant important, marqué par la reprise de l'autonomie industrielle et plus généralement économique par les Chinois.

6 Le passé de Tianjin comme microcosme dû à la présence de différentes nationalités lui a légué une richesse patrimoniale du point de vue matériel, comme les constructions encore visibles dans les anciennes concessions, et immatériel, comme les connaissances 
techniques acquises depuis plus d'un siècle. Bien qu'il soit porteur d'une empreinte du passé, le patrimoine est un sujet d'actualité. Face à la tension d'une urbanisation rapide, Tianjin doit décider que faire de son patrimoine. C'est dans ce contexte qu'est née la recherche académique entre l'Université de Tianjin et l'Université Paris 1 Panthéon Sorbonne qui couvre trois axes: la mondialisation, les transferts technologiques et la valorisation patrimoniale.

7 À l'issue de mon master Erasmus Mundus en Techniques, Patrimoine, Territoires Industriels, ma directrice de thèse, le Professeur Anne-Françoise Garçon, m'a orientée sur ce cadre de recherche collaborative. Contextualisée dans le tissu urbain, la société et le cadre juridique, l'étude portant sur les friches industrielles est plus susceptible de nourrir des projets de reconversion en faveur du développement local. Une discipline jeune en Chine, la recherche sur le patrimoine industriel, suscite autant de passion qu'elle présente de lacunes, notamment dans la méthodologie, en particulier du fait de l'absence de l'archéologie industrielle et de l'histoire des techniques. J'ai ainsi cherché à évaluer le rôle de l'histoire des techniques dans la révélation de la valeur patrimoniale des sites industriels en Chine.

8 L'origine occidentale, la production de l'électricité dans cinq centrales électriques distinctes, leurs transformations complexes au fil du temps, ont compliqué la recherche. La consultation de sources d'archives abondantes mais éparses en Chine et dans les pays étrangers s'est avérée être la première difficulté. La disparition progressive des vestiges de l'ancien réseau électrique du fait de l'urbanisation rapide en a été la seconde et elle est incontestablement la plus délicate. J'ai exploité trois fonds d'archives, en France, en Belgique et en Chine pour, en définitive, me concentrer sur trois compagnies: l'Énergie électrique de Tientsin, la Compagnie de Tramways et d'Éclairage de Tientsin, et la compagnie japonaise créée en 1938.

9 Ce choix archivistique est justifié par les raisons suivantes : l'Énergie électrique et la Compagnie de Tramways et d'Éclairage eurent une influence plus grande du fait de leur durée d'activités et de leur capacité de production. Après la guerre, en 1945, elles ont été nationalisées par le gouvernement chinois. Par ailleurs, la Compagnie de Tramways et d'Éclairage a été la seule compagnie qui a exploité les trois domaines - le tramway, l'éclairage électrique et la force motrice. Quant à la compagnie japonaise, inaugurée au début de la guerre, elle a annexé les autres centrales électriques et étendu le réseau dans la région, favorisant ainsi une industrialisation d'envergure. Elle a préparé à sa manière la transition vers le monopole étatique dans l'électrification qui a ensuite été adopté comme stratégie d'industrialisation par les Chinois entre 1945 et 1960.

Chaque fonds d'archives possède des atouts et des inconvénients pour la recherche thématique. Les archives diplomatiques, à Nantes, concernant la compagnie l'Énergie électrique, sont fragmentaires. Faute de pouvoir suivre son développement depuis sa création jusqu'à sa nationalisation, j'ai choisi de repérer les changements importants au fil du temps comme, par exemple, sa transformation de régie en compagnie privée et ses relations avec les autorités locales. En revanche, les archives de la Compagnie de Tramways et d'Éclairage sont complètes et abondantes. Les défis étaient donc la sélection et le tri des informations selon ma thématique. Je me suis efforcée d'étudier les archives qui révélaient sa suprématie technologique et les réseaux développés avec sa holding, ses fournisseurs, ses concurrents et les autorités locales. Les archives municipales de Tianjin conservent plutôt les dossiers relatifs à la gestion des centrales électriques par les Chinois après leur nationalisation. Malheureusement, les dossiers 
concernant l'électricité étaient mélangés ou mal classés, de sorte que je me suis concentrée sur la méthode des Chinois, telle que reflétée par les mesures prises pour réaliser une gestion efficace des centrales électriques disparates et séparées.

11 En ce qui concerne l'état actuel des vestiges électriques et de la patrimonialisation, j'ai visité les sites existants constitutifs de l'ancien réseau électrique: le bâtiment des bureaux de la Compagnie de Tramways et d'Éclairage et la centrale construite par les Japonais, en service jusqu'en 2011. L'analyse du tissu urbain environnant et des réalisations de mise en valeur du patrimoine bâti m'ont permis d'en constater les effets positifs et négatifs ainsi que les écarts entre la réglementation et la pratique.

12 Sources et terrain m'ont conduite à définir ma problématique. Pour la première phase d'électrification, de 1900 à 1937, j'ai cherché à examiner la capacité d'adaptation des compagnies étrangères d'électricité à Tianjin comme ville de concessions. Comment ont-elles fait face à la xénophobie des Chinois et à la concurrence avec les autres puissances étrangères? Quels étaient les avantages et inconvénients des différents modes d'organisation des compagnies? Comment chacun des trois domaines de l'électrification, la traction, l'éclairage électrique et la force motrice, a-t-il interagi avec l'urbanisation et l'industrialisation? Pour la deuxième phase, de 1937 à 1960, j'ai examiné la tendance à l'intégration des différents réseaux au service de l'industrialisation et la réussite de leur gestion par les Chinois. Comment les deux gouvernements chinois, les nationalistes et les communistes, se sont-ils distingués de leurs prédécesseurs dans la gestion du réseau électrique? Quels étaient les liens entre l'éducation technique à l'époque moderne et l'acquisition de capacités d'innovation technologique par les Chinois qui ont contribué à la réussite de l'électrification? En ce qui concerne le devenir des vestiges de l'électrification, j'ai remis en cause une politique d'aménagement urbain faisant de leur usage fonctionnel et de la valeur économique une priorité plutôt que l'aspect historique et la valeur culturelle. Pourquoi le cadre juridique de la conservation du patrimoine, fondé sur les théories occidentales et ayant évolué pendant un siècle, n'arrive-t-il pas à réguler le traitement du patrimoine bâti ? Comment les nouvelles approches et les pistes de recherche et de valorisation ont-elles changé la situation des friches industrielles en Chine?

$\mathrm{Au}$ final, quels sont les résultats principaux de cette recherche?

Premièrement, la situation délicate de Tianjin en raison des conflits et collaborations liés à son statut de ville de concessions, a eu pour effet d'accélérer les innovations et les appropriations techniques. En conséquence, les étrangers ont cherché à adapter leurs réalisations techniques et l'exploitation du réseau au marché local, et les Chinois se sont efforcés de s'acclimater à un nouvel ordre économique et technique mondial. Les compagnies étrangères d'électricité ont agi au niveau tant international que local pour organiser l'implantation, la direction et le financement, afin d'assurer un service local favorable. La Compagnie de Tramways et d'Éclairage, d'origine belge, a réussi non seulement à jouir du monopole du tramway, mais aussi à étendre sa zone de service grâce au modèle de holding. En effet, la forme de holding était apte à mobiliser au plan international les ressources financières et humaines ainsi que les relations diplomatiques. Par contre, l'Énergie électrique est tombée sur la coupe de la concession française du fait des difficultés de financement et des litiges avec d'autres compagnies.

Deuxièmement, les trois domaines de l'électrification se sont développés à mesure que Tianjin s'urbanisait et s'industrialisait. En favorisant le déplacement d'une population plus large entre la cité chinoise et les concessions étrangères, le tramway a connu une 
forte croissance entre 1910 et 1915. Malgré la stagnation du réseau à partir des années 1920, le tramway a cependant accéléré l'urbanisation des concessions française et japonaise, qui ont remplacé la cité chinoise, pour devenir le centre commerçant et financier. Quant à l'éclairage électrique, il a poussé les centrales électriques à augmenter leur capacité de production et à organiser leur mise en réseau. Malgré un développement accéléré, l'utilisation de l'électricité en tant que force motrice était toujours limitée par le sous-développement de la production mécanique avant 1937.

16 Troisièmement, au moment de l'occupation japonaise, l'industrialisation, celle de la région, a eu pour effet l'intégration des centrales et l'extension du réseau au nord de la Chine, ce qui a mis fin au monopole européen et conduit au monopole étatique. L'attitude du gouvernement chinois, lorsqu'il a repris les centrales électriques, a été d'adopter une méthode rationnelle pour leur exploitation. Les communistes sont allés plus loin en redéfinissant l'électrification comme moteur de l'industrialisation et l'ont adaptée à la planification quinquennale. À défaut d'investissements, les Chinois ont activement recouru à des innovations technologiques pour résoudre la tension entre la demande et la fourniture d'électricité.

Quatrièmement, l'introduction de l'éducation technique occidentale et son adaptation à l'industrialisation en Chine ont contribué à l'implantation de métiers spécialisés et qualifiés pour les postes de techniciens et de gestionnaires. L'action des fonctionnaires créateurs des institutions modernes vouées à la diffusion des connaissances techniques n'était que la continuation de l'ancien système de formation aux techniques artisanales, qui représente le régime de la technique. Plus tard, ce sont les initiatives privées et ensuite les efforts collectifs des ingénieurs chinois qui ont orienté l'éducation vers une professionnalisation plus adaptée au développement industriel. La réforme a finalement séparé les techniciens des fonctionnaires, qui avaient l'apanage du savoir technique, et formé les techniciens pour mettre en œuvre des innovations technologiques. L'électrification a ainsi bénéficié des moyens d'apprentissage pour la formation des ingénieurs et ouvriers capables d'appliquer leurs acquis à la situation spécifique en Chine.

Cinquièmement, la notion du patrimoine industriel vient de l'Occident. Son adoption en Chine nécessite une appropriation tenant compte des particularités locales. La priorité qui a été donnée aux objectifs économiques à court terme plutôt qu'à la conservation des témoignages historiques a engendré un manque d'observations de la réglementation. La perception traditionnelle mettant l'accent sur les sources écrites plutôt que le bâti et le mépris de certains types de patrimoine exercent toujours un impact négatif sur le choix de classement et les méthodes de conservation et de restauration. Que ce soit pour les sites étrangers ou chinois, la valeur patrimoniale est le point de départ pour classer un site et concevoir un projet de valorisation. En reconnaissant désormais la valeur de son patrimoine pour illustrer son histoire et son potentiel pour une relance économique, Tianjin a activement poussé la patrimonialisation des friches industrielles, qui pourrait rejaillir sur l'ancien réseau électrique.

Quels sont les prolongements possibles de ces recherches à l'avenir ? Il conviendrait de comparer l'électrification de Tianjin avec celle d'autres villes chinoises et étrangères, notamment des pays qui ont installé les centrales à Tianjin, pour faire ressortir ses caractéristiques uniques. Cela permettrait de poursuivre l'étude sur l'expansion en Extrême-Orient des compagnies holdings faisant notre sujet: la Compagnie 
Internationale d'Orient et la Banque de l'Indochine qui se sont implantées à Shanghai, Hong-Kong, Singapour, etc. La question du transfert technique entre l'Occident et la Chine nous invite à examiner la scène de collaboration entre les ingénieurs tant étrangers que chinois et les ouvriers afin de révéler une application des connaissances théoriques dans la pratique. Pour comprendre la prise de conscience des ingénieurs chinois en faveur d'une réforme de l'éducation, nous pourrons comparer les parcours et les activités des ingénieurs chinois ayant reçu leur formation à l'étranger et ceux proposés par les universités chinoises. Cette piste nous conduirait à associer leur perception des différences entre les pays développés et la Chine et les mesures pour adapter l'apprentissage au terrain de la pratique. En ce qui concerne la patrimonialisation des vestiges industriels, il serait opportun d'étudier le manque d'intérêt des entreprises pour valoriser les friches industrielles à des usages économiques autres que l'immobilier et le tertiaire. Nous nous interrogerons sur les moyens d'évaluer les effets tant positifs que négatifs à long terme des projets de reconversion.

Mon cheminement doctoral a été un transfert technique consistant à appliquer des théories étrangères pour étudier la situation chinoise. Dans la pratique, avant de découvrir le grand décalage entre les normes académiques chinoises et françaises, ma pensée chinoise a continué à régner. C'est la prise de conscience qui constitue le premier pas d'une nouvelle recherche d'apprentissage et d'appropriation. Il en va de même pour le transfert technique dans l'industrialisation datant du début du $\mathrm{XX}^{\mathrm{e}}$ siècle et dans la patrimonialisation aujourd'hui.

21 Monsieur le Président, Mesdames et Messieurs, membres du jury, je vous remercie de votre attention.

\section{RÉSUMÉS}

Tianjin s'est avérée un lieu d'échanges entre les Chinois et les étrangers pendant plus de 150 ans. Face au risque d'effondrement du pays, les pionniers chinois ont continué leurs essais de modernisation à Tianjin pour obtenir l'autonomie en termes industriels et techniques. Aujourd'hui, confrontée à l'urbanisation destructrice, la municipalité de Tianjin cherche activement à sauvegarder son patrimoine lié au passé et significatif pour le futur. A travers une étude sur l'électrification de Tianjin et sur la conservation de ses vestiges, cette thèse montre qu'il existe un point commun à ces deux actions à deux époques différentes : l'apprentissage de connaissances modernes auprès de l'Occident et leur appropriation en Chine qui donne une force de renouvellement à ce pays de culture très ancienne. L'électrification de Tianjin est un cas d'étude qui nous permet d'analyser le passage du contrôle absolu par les étrangers à la gestion réussie par les Chinois. Nous étudierons les conflits entre la technicité de l'électrification extension du réseau - et les services séparés dans les différentes zones de la ville administrées par plusieurs municipalités. Nous traiterons ensuite de l'intégration progressive et de la gestion systématique $\mathrm{du}$ réseau électrique sous le monopole étatique chinois après la fin de l'impérialisme en Chine. L'urgence de la conservation des friches industrielles et la multiplication des pôles créatifs - forme qu'elles prennent après reconversion - constituent notre point de 
départ pour questionner l'efficacité du cadre juridique adopté pour le patrimoine et ses améliorations depuis un siècle. Nous fondant sur la politique patrimoniale de Tianjin, nous explorerons les possibilités et les moyens efficaces de valoriser de nos jours son ancien réseau électrique.

Tianjin has been such a place where Chinese and the foreigners exchanged frequently for over 150 years. Facing the risk of a dissembling country, the Chinese pioneers continued to lead the modernization in order to achieve industrial et technical autonomy. Today, confronted to the crisis of identity lost, the municipality of Tianjin strives to protect its own heritage, inherited from the past and is significant for the future. Based on a study of the history of electrification and of the conservation of the vestiges, this dissertation shows the common point between these two actions in two periods: to learn modern knowledge from the western world and apply it to China. This application gives the country with an ancient culture the full energy to rejuvenate. Electrification appears a case study for analyzing the transformation from the total foreign control and operations to the successful management by the Chinese. We will study the conflicts between the technical requirement of electrification, which is the network extension, and the divided service in different zones of the city administrated by various municipalities. We will further explore the integration of these separate networks and their systematic management under the state monopoly after imperialism ended in China. The urgent task of industrial brownfields conservation and their transformation into hubs for creative activities raise an issue for us to look into the effectiveness of the legal framework for heritage protection and its continued improvements during one century. Based on the politics of Tianjin for heritage, we will explore the possibilities and sound ways of enhancing the value of its old electric network.

\section{INDEX}

Mots-clés : histoire des techniques, électricité, patrimoine industriel, friche industrielle, ville, industrialisation, politique urbaine, ville coloniale

Keywords : history of technology, town, urban planning, electricity, industrial heritage, industrialization, colonial town, heritage

Thèmes : Positions de thèse

\section{AUTEUR}

\section{JIALI HUANG}

Jiali Huang est docteure en Histoire des Techniques. Sa thèse s'intitule « Le réseau d'électricité de Tianjin (Chine), de 1900 à 1960. Histoire et valorisation patrimoniale ». Ses recherches portent sur l'électrification de Tianjin en Chine, initiée dans les concessions étrangères de la ville puis transformée et développée par les Chinois. Elle y analyse l'interaction entre les différentes cultures techniques. Elle examine ensuite les possibilités de valorisation du réseau électrique en tant que patrimoine industriel dans un contexte de multiplicité patrimoniale et d'urbanisation « destructrice ». Ses axes de recherche s'élargissent aux innovations techniques de la Chine moderne dans le contexte des échanges Orient-Occident, les politiques et les pratiques de conservation et valorisation du patrimoine en Chine, et la valorisation des savoir-faire techniques en faveur de la compétitivité des villes chinoises. Elle a publié un article sur la situation déséquilibrée de réutilisation et conservation du patrimoine industriel en Chine, et dirigé le numéro « Ville et technique », dans la revue e-Phaistos. Un autre article sur le développement du réseau du tramway à Tianjin avant 1937 sera publié prochainement. 